\title{
Fast Power- and Slew-Aware Gated Clock Tree Synthesis
}

\author{
Jingwei Lu, Wing-Kai Chow, and Chiu-Wing Sham
}

\begin{abstract}
Clock tree synthesis plays an important role on the total performance of chip. Gated clock tree is an effective approach to reduce the dynamic power usage. In this paper, two novel gated clock tree synthesizers, power-aware clock tree synthesizer (PACTS) and power- and slew-aware clock tree synthesizer (PSACTS), are proposed with zero skew achieved based on Elmore RC model. In PACTS, the topology of the clock tree is constructed with simultaneous buffer/gate insertion, which reduces the switched capacitance. In PSACTS, a more practical clock slew constraint is applied. Compared to previous works, clock tree synthesis is done first and followed by the insertions of clock gates. The clock slew changes a lot after the insertions of clock gates in real cases. In our work, the clock tree is constructed simultaneously with the insertions of clock gates. This ensures the limitation of the clock slew can be strictly satisfied while the limitation of the clock slew is always applied in the real design. The experimental results show that the power cost of our work is smaller and the runtime is reduced. The slew rate constraint is satisfied with a small clock skew from SPICE estimation. Generally, our work has better performance, improved efficiency and is more practical to be applied in the industry.
\end{abstract}

Index Terms - Clock gating, clock tree synthesis, design automation.

\section{INTRODUCTION}

C LOCK signals are employed in VLSI digital systems to synchronize the active components. A clock tree is constructed to deliver the synchronous signal to every sequential cell of the digital circuits. The clock skew represents the timing difference of all terminals in the clock tree. In order to synchronize one circuit, each terminal must be reached within a specified small time range or a bounded skew [1]. Additionally, the clock signal is also the single largest source of dynamic power usage in the system. There is around $30 \%$ to $50 \%$ of the chip power which is consumed by the clock tree [2]. Owing to the high frequency of switching as well as the expansion of the clock tree over the whole chip, a huge amount of energy dissipation is consumed by the clock tree. Based on the concept of the low power design, especially for the mobile equipments, the improvement on the clock power consumption is crucial.

Manuscript received August 16, 2010; revised January 09, 2011 and May 24, 2011; accepted September 08, 2011. Date of publication October 17, 2011 date of current version July 27, 2012. This work was supported by a grant from the Research Grants Council of the Hong Kong Special Administrative Region, China (Project No. PolyU 5263/10E).

The authors are with the Electronic and Information Engineering, The Hong Kong Polytechnic University, Hong Kong (e-mail: francesco.ljw@gmail.com; williamchowhk@gmail.com; encwsham@polyu.edu.hk).

Color versions of one or more of the figures in this paper are available online at http://ieeexplore.ieee.org.

Digital Object Identifier 10.1109/TVLSI.2011.2168834
Clock skew minimization is a popular research topic during the past decades. Plenty of research achievements have been proposed by previous researchers. Some earlier proposed works concentrated on the distribution of wirelength between the source and the sinks to achieve the linear delay equalization. Jackson [3] first presented a clock routing algorithm based on a sub-optimal equidistant network. A top-down recursive horizontal by vertical partitioning was constructed. Later, more improvements were made to generate clock tree of exactly equidistant path-length [4]. A bottom-up geometric matching was then proposed [5], with zero linear clock skew. Afterwards, the delay balancing technique using Elmore RC model [6] became prevalent to reach exact zero skew [7]. The deferred-merging and embedding (DME) technique [8], [9] was proposed to achieve zero skew with minimal wirelength by segments. While the scale of the clock tree becomes larger and larger, buffer insertion was also considered in clock tree synthesis [10], [11]. Van Ginnecken proposed a buffer insertion approach [10] with time complexity $O\left(N^{2}\right)$. This was later improved in Shi et al. [12]. Some researchers minimized the number of buffer insertions in clock tree to reduce power consumption [13], [14] and phase delay [11]. Efforts have been made to improve clock tree topology. Edahiro [15] proposed a clustering-based topology generation in $O(N \log N)$ for linear clock routing. Topology design in the buffered clock tree was discussed in [16]-[19].

The application of the gate insertion is an effective approach to reduce the power usage. The principal idea is to turn off the clock signal of the idle circuits. The unnecessary switching power caused by the clock signal can be reduced in this way. The clock gating technique can be applied on the logic level [20], register-transfer-level (RTL) [21], and architecture level [22], respectively. A deterministic clock gating technique is proposed [23] based on the actual usage of circuit blocks. It can reduce the power consumption of microprocessors. Optimization on upper design levels may not consider the physical information and it causes unnecessary detours or snaking wires. The improvement on power saving by applying the clock gating technique on the logic level is usually small [24]. In fact, the physical location of the logic components should be taken into account during the clock gating design. Recently, a gated clock design was proposed to optimize the performance based on the appropriate placement of clock gates [25]. With the assistance of the physical information of the modules, wirelength overhead of the clock tree can be improved with reduction on the power usage.

In the past, some achievements regarding the clock gating design have been proposed. Both the logical and physical concerns are considered in these research works. The design of an ac- 
tivity-driven gated clock tree was proposed [26], [27]. However, clock skew was only handled by balancing the gate number. The contribution of wires was neglected. It means that the gate insertion was not applied concurrently within the topology generation. The similarity of activity patterns between the clock nodes [28] was proposed to be utilized. The performance was improved with reduced power consumption. An instruction stream [29] was proposed later to simulate the probabilistic information of each module, and a switched capacitance is used to denote the power consumption of the clock tree. Two clock gating methods [30] regarding microprocessor design, GCR and GCRred, was developed to minimize the switched capacitance. However, no relationship of activities was concerned while merging each pair of nodes. The resulted clock tree was still non-zero skew.

Recently, a comprehensive technique [31] was proposed. It is composed of a recursive computation on effective switched capacitance and a solution sampling method based on merging segment set. Two according methods, GBCR and GBCRnt, were introduced and analyzed. Despite the improvement on performance, the approach consumes too much time and memory. The slew rate is assumed to be an ideal situation with limitation on the total downstream capacitance only which may not be practical in real cases. They assumed that the clock gates have the same physical characteristics as the buffers so they can simply replace the buffers by the clock gates if needed. Practically, the physical characteristics of the buffers are not identical to the clock gates. Simple replacement may cause the violation of clock slew and a significant increment of clock skew. While the process of clock tree synthesis and clock gates insertion are executed concurrently, the position of clock gates can be determined precisely to maintain the clock skew and clock slew.

In this paper, we propose two novel gated synthesizers, power-aware clock tree synthesizer (PACTS) as well as powerand slew-aware clock tree synthesizer (PSACTS). A binary clock tree is constructed in a bottom-up course. In PACTS, we apply the same slew constraint as those previous clock gating works [30], [31]. Based on the nearest-neighbor-selection (NNS) [15] binary tree topology construction, we propose a novel simultaneous gate/buffer insertion method to reduce the power. The number of gates/buffers and the wirelength of enable signals can be effectively controlled. Meanwhile, we propose an enhancement to update the activity of internal nodes with reduced complexity, which improves the program efficiency. The experimental results show that our method consumes less power compared to all the published research works in the literature. The runtime of our program is also the smallest because of reduced complexity. In PSACTS, a more strict slew constraint, such that the clock slew should be less than $100 \mathrm{ps}$ [32], is employed along the whole clock tree. We propose a slew-oriented lookup table to provide the information of driving ability during the buffer and gate insertion. The experimental results also show that the slew rate limitation can be satisfied with a small clock skew from SPICE estimation. It shows that our work has better performance, improved efficiency and becomes more practical to be applied in the industry.

The remainder of this paper is organized as follows. Section II includes the problem formulation. Section III introduces the

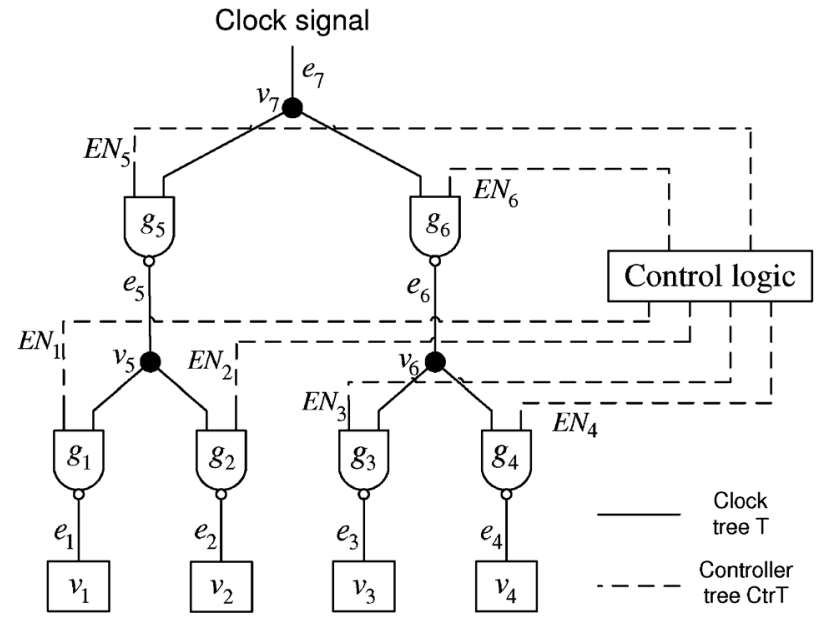

Fig. 1. Gated clock tree (binary tree).

major contribution of our two synthesizers: the concurrent gate/ buffer insertion, the fast information updating and the slew table construction. In Section IV, we present our experimental results based on two well-known benchmark suites. Finally, the conclusion is given in Section V.

\section{Problem Formulation}

In this section, some basic concepts regarding our research work are introduced first. It includes the general definition of clock tree, controller tree, the activity of each node, slew constraint, RC models of buffers, clock gates and wires and switched capacitance. The specific problem formulation can be found at the end of this section.

\section{A. Clock Tree and Controller Tree}

Let $T$ denote the clock tree. The set of leafs (sinks), nodes and edges are denoted by $S, V$, and $E$, respectively. $|S|,|V|$, and $|E|$ become the number of elements of $S, V$, and $E$, respectively. Hence, we have $T=\{V, E\}$ and $|E|=|V|-1$. $E=\left\{e_{j}|j=1,2, \ldots| V \mid-1,\right\}$, where $e_{j}$ is the edge between $v_{j}$ and its corresponding parent. Let $d_{s_{i}}$ denote the signal delay of the $i$ th leaf. The rest $(|V|-|S|)$ nodes are named as internal nodes. The leaves are at level 0 . The root is at the highest level. Node $v_{i}$ is said to be at the $j$ th level if there are $j$ edges on the path from $v_{i}$ to the furthest leaf of $T$. We assume that the topology of the clock tree is binary. It means that every internal node has exactly two children. The skew of $T$ is the difference between the longest signal delay and the shortest signal delay from the root to any leaves of the clock tree. The control logic is assumed to be located at the center of the chip [30], [31]. The controller tree is denoted as $T^{\text {ctr }}$ with the application of star routing. A control edge $E N_{i}$ in $T^{\mathrm{ctr}}$ transmits the enable signal to the respective gate $g_{i}$ on the edge $e_{i}$ in the clock tree $T$. An example of a clock tree $T$ and its controller tree $T^{\mathrm{ctr}}$ is shown in Fig. 1. In this paper, sink, leaf, module, and flip-flop all denote sink node of a clock tree.

During the operating time of a circuit, each flip-flop of the circuit (module) has its active time and idle time. It is usually specified as different activity patterns. The modules are the sink nodes of the clock tree. The activity patterns of the nodes can be 


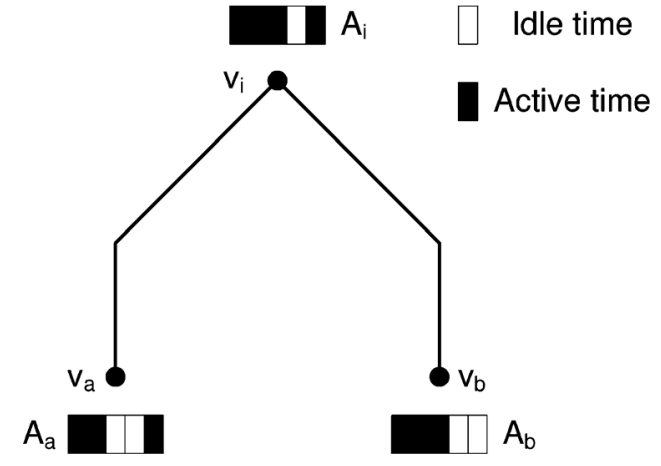

Fig. 2. Bottom-up activity pattern transmission.

obtained by the simulation of the design at the behavioral level [27]. Let $A_{i}$ be the activity pattern of the node $v_{i}$. It is a binary string with " 1 "s indicating the active periods and " 0 "s indicating the idle periods of a sink or an internal node. If $v_{i}$ is a sink node, we can directly obtain $A_{i}$ from the benchmark file. Otherwise, $v_{i}$ is supposed to be an internal node with two children nodes $v_{L}$ and $v_{R}$. The clock signal at $v_{i}$ must be enabled whenever its left or right child is active. Therefore, $A_{i}$ is calculated by performing the bitwise $O R$ operation on the activity patterns of $v_{L}$ and $v_{R}$. Hence, $A_{i}$ equals $A_{L} \cup A_{R}$. An example of a bottom-up activity pattern transmission is shown in Fig. 2.

Let $p_{i}$ denote the activity of the node $v_{i}$, and $p_{i}^{t r}$ denote its transition probability. These two factors are calculated based on $A_{i}$. The specific equations are shown as follows:

$$
p_{i}=\frac{A T_{n o}\left(A_{i}\right)}{\operatorname{Len}\left(A_{i}\right)}, \quad p_{i}^{t r}=\frac{T R_{n o}\left(A_{i}\right)}{2 \times\left(\operatorname{Len}\left(A_{i}\right)-1\right)}
$$

where $A T_{n o}\left(A_{i}\right)$ is the number of active times (" 1 "s) in $A_{i}$, and $T R_{n o}\left(A_{i}\right)$ is the number of transitions (" 10 " or " 01 ") in $A_{i}$. Len $\left(A_{i}\right)$ denotes the stream length of $A_{i}$.

\section{B. Slew Rate Constraint}

The restriction on clock slew describes the requirement on the signal transition time reduction. The clock slew is defined to be the rising time from $10 \%$ to $90 \%$ of the signal strength $(90 \%$ to $10 \%$ for the falling time). In common practice, the upper limit is set to be 100 ps [32]. During the clock tree synthesis, it is necessary to maintain the signal transition time below this upper limit throughout the whole tree. Otherwise, the slew violation occurs and the clock tree solution fails.

\section{RC Model of Interconnect Wires, Buffers, and Clock Gates}

The RC models of an interconnect wire, a buffer and a clock gate are shown in Figs. 3 and 4, respectively. In Fig. 3, the length of the wire is denoted as $L$. The unit capacitance and unit resistance of the wire are denoted as $C_{w}$ and $R_{w}$. In Fig. $4, d_{b}$ is included to indicate the intrinsic delay of a buffer (gate). Intrinsic delay $d_{b}$ is assumed to be constant in our simplified RC model. This assumption is appropriate because the clock slews are always maintained at around 80 to $100 \mathrm{ps}$ with our table-lookup approach of the buffer/clock gate insertion. $C_{b}\left(C_{g}\right)$ denotes the input capacitance and $R_{b}\left(R_{g}\right)$ denotes the driver resistance of a buffer (gate). Wires, buffers and clock gates are dominant factors of the total capacitance and the clock phase delay. Clock

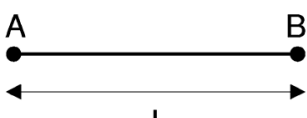

L

(a)

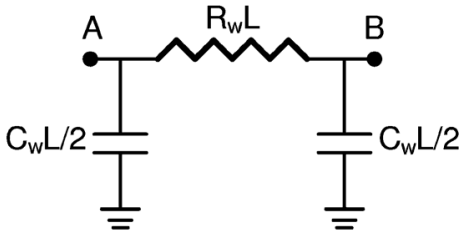

(b)
Fig. 3. RC model of interconnect wire. (a) A segment wire. (b) An equivalent model.

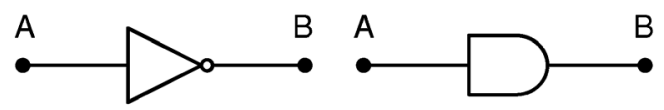

(a)

(b)

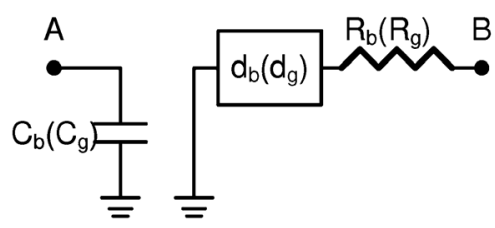

(c)

Fig. 4. RC model of buffer and clock gate. (a) A buffer. (b) A clock gate. (c) An equivalent circuit model.

signals will be inverted by buffers, so the two clock signals at a merging point should be maintained at the same polarity. We use AND or NAND gates as the clock gate in our approach depending on the parity of branches but both of them have the same RC model. Notice that the RC model is utilized only for delay computation during the clock tree synthesis. During the slew-table construction, we use the transistor-level models proposed in ISPD contests for the clock slew estimation.

\section{Switched Capacitance}

The power consumed by CMOS circuits consists of two components: static power and dynamic power. The static power is mostly determined by the feature size and other technologies. Therefore, we only consider dynamic power minimization in this paper. The definition of the dynamic power is shown as follows:

$$
P=\frac{1}{2} \alpha C f V_{d d}^{2}
$$

where $C$ means the total load capacitance on the circuit, $f$ is the frequency of the clock signal and $V_{d d}$ is the power supply. $\alpha$ means the amount of switching times of the corresponding signal in each clock cycle. For the clock tree, $\alpha$ should be two because the clock signal has one rising and one falling edge in each clock period. Alternatively, $\alpha$ should be one in the controller tree. Since $f$ and $V_{d d}$ are constant parameters in the digital circuits, we can use the switched capacitance $(S C)$ as a measure of the power usage. It indicates the active capacitance in average of one clock period and it is commonly used in most previous works [30], [31].

An example is shown in Fig. 5. Let $v_{a}$ be the currently concerned node. $v_{x}$ and $v_{y}$ are two children nodes of $v_{a} . v_{u}$ is the parent node of $v_{a} . v_{a}$ is connected to its parent node $v_{u}$ by the edge $e_{a} . v_{x}$ is connected to its parent node $v_{a}$ by the edge $e_{x} . v_{y}$ is connected to its parent node $v_{a}$ by the edge $e_{y}$. Let $C_{a}^{u}$ denote 


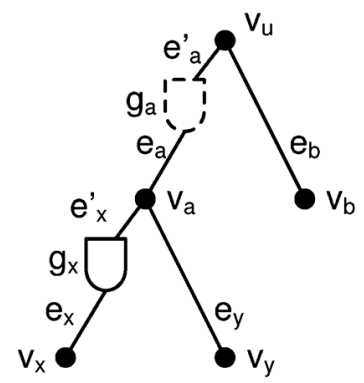

Fig. 5. Binary clock tree with clock gate insertion.

the masked downstream capacitance for node $v_{a}$. A clock gate $g_{x}$ is inserted on the edge $e_{x}$. In Fig. 5, $C_{a}^{u}$ is denoted by the following formula:

$$
C_{a}^{u}=C_{g}+\left(\left|e_{x}^{\prime}\right| \times C_{w}\right)+\left(\left|e_{y}\right| \times C_{w}+C_{y}^{u}\right) .
$$

As the clock gate is always inserted at the top end of the edge, $\left|e_{x}^{\prime}\right|$ is zero in this case. Thus, $C_{a}^{u}$ is computed by the following equation:

$$
C_{a}^{u}=C_{g}+\left(\left|e_{y}\right| \times C_{w}+C_{y}^{u}\right) .
$$

If a gate $g_{a}$ is inserted on the edge $e_{a}\left(\left|e_{a}^{\prime}\right|\right.$ is always zero), there should be a controller edge for the enable signal transmission of $g_{a}$. This edge is denoted by $e_{a}^{\text {ctr }}$. It connects the gate control logic to the enable/disable input of the clock gate. For the edge $e_{a}^{\text {ctr }}$, the load capacitance and the corresponding switched capacitance are defined by the following equations:

$$
C_{a}^{\mathrm{ctr}}=\left|e_{a}^{\mathrm{ctr}}\right| \times C_{w}+C_{g} .
$$

Finally, the resultant switched capacitance at node $v_{a}$ if there is a clock gate that is inserted on $e_{a}$ (gated) is computed by the following equation:

$$
S C_{a}=\left(C_{a}^{u}+\left|e_{a}\right| \times C_{w}\right) \times p_{a}+C_{g}+C_{a}^{\mathrm{ctr}} \times p_{a}^{t r}
$$

where $p_{a}$ is the activity of $v_{a}$, and $p_{a}^{\text {ctr }}$ is the transitional probability of $v_{a}$.

If there is no clock gate inserted on $e_{a}$ (non-gated), the resultant switched capacitance at node $v_{a}$ is computed by the following equation:

$$
S C_{a}=C_{a}^{u}+\left|e_{a}\right| \times C_{w} .
$$

In general, the resulting total switched capacitance $S C_{a}$ is contributed by $v_{a}, e_{a}$, and $e_{a}^{\text {ctr }}$. If the switched capacitance $\left(S C_{a}\right)$ of (6) is smaller than that of (7), the power usage can be reduced. Since our clock tree synthesis is in a bottom-up procedure of clock routing, $S C_{a}$ cannot be changed by any subsequent gate insertion at upper levels if $g_{a}$ is already inserted.

Given the physical location and the logic information, which denotes the activity patterns of the modules, the objective of our work is to construct a buffered and gated clock tree and the corresponding controller tree. Subject to the two constraints of zero skew and slew rate, the total switched capacitance that is contributed by both the clock tree $T$ and the controller tree $T^{\mathrm{ctr}}$ should be minimized.

\section{METhOdOLOGY}

In this work, two novel clock tree synthesizers, PACTS and PSACTS, are proposed to improve the clock skew and reduce the power consumption. Our synthesizers are designed in a bottom-up procedure to construct a buffered and gated binary clock tree. Some traditional techniques are applied in our synthesizers. The deferred-merging and embedding (DME) technique [8], [9] is applied for the wirelength minimization. The NNS technique [15] is applied for topology generation and Elmore model [6] is applied for delay computation.

PACTS uses the same slew constraint as many previous clock gating works [30], [31]. Buffers and gates are assumed to be of the same sizes, and they are inserted at the top end of every edge (same as [30]). Thus, the reduction of switched capacitance can be maximized. The slew constraint is defined as that the driver capacitance of each buffer/gate cannot exceed 20 times of its input capacitance. We compute the merging cost of each node pair, assuming to be $v_{a}$ and $v_{b}$, with the following equation:

$$
f_{a, b}=\left|e_{a}\right| \times p_{a}+\left|e_{b}\right| \times p_{b}
$$

where $\left|e_{a}\right|$ and $\left|e_{b}\right|$ denote the length of edge, and $p_{a}$ and $p_{b}$ are the activity, accordingly. NNS is applied to merge the pair with minimum cost every time, in which we construct the topology. We propose a simultaneous gate/buffer insertion method to improve the switched capacitance of the clock tree, as described in Section III-A. This can help to reduce the gate usage and scale down the controller tree. Additionally, we propose a fast information updating method to reduce the complexity of our program, as described in Section III-C. The efficiency of the work is improved accordingly. PSACTS is constructed based on PACTS, which employs a more strict constraint on clock slew such that the clock slew should be less than 100 ps [32]. A lookup table is developed as a slew rate reference. During the synthesis, we propose a fast and accurate approach to determine the driving ability of each buffer and gate according to the lookup table. This method is discussed in Section III-B. We analyze the complexity of all the gated clock works in Section III-C.

In the following sections, the methodologies of our synthesizers are discussed in details.

\section{A. Concurrent Gate Insertion}

We propose a new method to determine the insertion of buffer/gate simultaneously. This method ensures that the slew constraint can be satisfied while there is an insertion of either a buffer or a clock gate. According to the slew constraint, it is required to insert a buffer or a clock gate when the downstream capacitance is over the limitation. Both the buffer and the clock gate can supply driving power to reduce the signal transition time and reduce the clock slew. If the downstream capacitance is over the limitation on edge $e_{a}$, a clock gate $g_{a}$ should be inserted on $e_{a}$ (the top end of $e_{a}$ ) to support the driving power (gated). The resulting switched capacitance becomes $S C_{a}^{g}$. If a buffer $b_{a}$ is inserted instead (buffered), the switched capacitance is changed to be $S C_{a}^{b}$. If there is no slew violation at $e_{a}$, we can connect $v_{a}$ with $v_{u}$ directly without the insertion of a buffer or a clock gate (non-gated and non-buffered). The resulting switched capacitance is $S C_{a}^{n}$. An example is shown in 


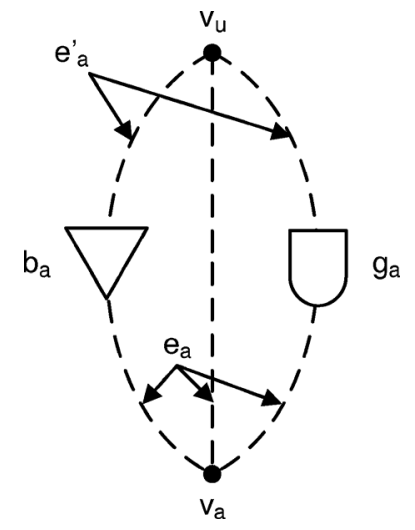

Fig. 6. Possible connection between $v_{a}$ and its parent $v_{u}$.

Fig. 6 to illustrate these three cases. The formulas to compute the resulting switched capacitances are shown as follows:

$$
\begin{aligned}
S C_{a}^{n} & =\left|e_{a}\right| \times C_{w} \\
S C_{a}^{b} & =S C^{n}+C_{b} \\
S C_{a}^{g} & =S C_{a}^{n} \times p_{a}+C_{g}-C_{a}^{u} \times\left(1-p_{a}\right)+C_{a}^{\mathrm{ctr}} \times p_{a}^{t r} .
\end{aligned}
$$

In order to determine whether a clock gate should be inserted for any new merging node $v_{u}$, we can compare the resulting switched capacitance of different conditions. If a smaller switched capacitance can be obtained, the total switched capacitance of the clock tree can be smaller accordingly. For simplicity, $S C_{a}^{v}$ is used to denote the condition that there is no insertion of clock gate. It means that $S C_{a}^{v}$ becomes $S C_{a}^{n}$ while there is no slew violation on edge $e_{a}$ and $S C_{a}^{v}$ becomes $S C_{a}^{b}$ while a buffer should be inserted to satisfy the slew constraint on edge $e_{a}$. The definition of $S C_{a}^{v}$ is shown as follows:

$$
S C_{a}^{v}= \begin{cases}S C_{a}^{n}: & \text { No buffer is required at } e_{a} \\ S C_{a}^{b}: & \text { A buffer is required at } e_{a} .\end{cases}
$$

In general, there are five possible conditions to determine whether a clock gate is required to be inserted on either branch $e_{a}$ or branch $e_{b}$ of the new merging node $\left(v_{u}\right)$.

1) No clock gate is inserted on both $e_{a}$ and $e_{b}$. The corresponding cost is denoted by $f_{a, b}^{N}$.

2) A clock gate is inserted on $e_{a}$ but no clock gate is inserted on $e_{b}$. The corresponding cost is denoted by $f_{a, b}^{L}$.

3) No clock gate is inserted on $e_{a}$ but a clock gate is inserted on $e_{b}$. The corresponding cost is denoted by $f_{a, b}^{R}$.

4) A clock gate is inserted on $e_{a}$ and a clock gate is inserted on $e_{b}$. The corresponding cost is denoted by $f_{a, b}^{D}$.

5) No clock gate is inserted on both $e_{a}$ and $e_{b}$ but a clock gate is inserted on $e_{u}$. The corresponding cost is denoted by $f_{a, b}^{V}$.

The equations to compute the corresponding cost values are shown as follows:

$$
\begin{aligned}
& f_{a, b}^{N}=S C_{a}^{v}+S C_{b}^{v} \\
& f_{a, b}^{L}=S C_{a}^{g}+S C_{b}^{v} \\
& f_{a, b}^{R}=S C_{a}^{v}+S C_{b}^{g} \\
& f_{a, b}^{D}=S C_{a}^{g}+S C_{b}^{g} \\
& f_{a, b}^{V}=f_{a, b}^{N} \times p_{u}+C_{g}-\left(C_{a}^{u}+C_{b}^{u}\right) \times\left(1-p_{u}\right)+C_{u}^{\mathrm{ctr}} \times p_{u}^{t r} .
\end{aligned}
$$

For $f_{a, b}^{V}$, a gate is only virtually assigned for $v_{u}$ at the upper level. Since the location of $v_{u}$ is not decided, the corresponding gate location can be assumed to be the same with $g_{a}$ and $g_{b}$. If $\min \left(f^{V}, f^{N}\right)$ is smaller than $\min \left(f^{L}, f^{R}, f^{D}\right)$, no gate is inserted on either $e_{a}$ or $e_{b}$. It can be easily proved that the switched capacitance is smaller without the gate insertion under these cases. If $f^{V}$ has the minimum value, the similarity of $A_{a}$ and $A_{b}$ is probably very large and $p_{u}$ is close to $p_{a}$ and $p_{b}$.

For each pair of nodes for merging, we compute all the cost values of above five categories by their corresponding configuration. The minimum value of these five can help us to determine whether the clock gate should be inserted on edge $e_{a}$ and $e_{b}$. The minimum value can be obtained by the following equation:

$$
f_{a, b}=\min \left(f^{N}, f^{L}, f^{R}, f^{D}, f^{V}\right) .
$$

The major target of our design is to minimize the total switched capacitance. Without the insertion of clock gate, the increment of switched capacitance in the merging is $S C_{a}^{n}$ (no slew violation on $e_{a}$ ) or $S C_{a}^{b}$ (buffer should be inserted to satisfy the slew constraint). The insertion of gate will not only cause a reduction of switched capacitance on the clock tree, but also an increment of switched capacitance on the controller tree. Thus, there is a tradeoff between these two. By finding the value of $f_{a, b}$, we can determine whether (and how) a clock gate should be inserted in order to construct a clock tree with minimum resulting switched capacitance.

\section{B. Slew Table Construction}

According to the slew constraint in [30], [31], it is required to insert buffer or clock gate when the downstream capacitance is over twenty times of the input capacitance of a single gate. Both buffer and clock gate can supply driving power to reduce the signal transition time and reduce the clock slew. If $C_{d}$ is larger than $20 \times C_{g}$ at the edge $e_{a}$, a clock gate or buffer should be inserted at $g_{a}$ (the top end of $e_{a}$ ) to support the driving power. However, downstream capacitance is not an accurate measurement to estimate the clock slew. In real VLSI design process, the hard slew rate constraint should be satisfied.

In real case, a concrete slew rate constraint should be applied on all clock tree branches in the clock tree synthesis. For example, $100 \mathrm{ps}$ is used in ISPD clock network synthesis contest [32]. However, real-time slew tuning costs a lot of time and this reduces the efficiency of the system. Instead of it, a slew table is proposed to be applied in PSACTS. A lookup table can be pre-constructed for slew reference by SPICE simulation before the execution of our program. The procedure to construct the slew table based on two categories: single wire and binary branches. At any node, we can obtain the maximum driving length from the slew table according to the upstream buffer size, the downstream buffer size and the downstream wirelength. An example is shown in Fig. 7. We can assume that the current node is $v_{0} . l_{0}$ is the maximum driving length for next buffer insertion $b_{0} . l_{0}$ is supposed to be obtained from the slew table in the program. $s z_{b_{0}}$ is the buffer size (a number of buffers connected in parallel). If it is a single wire connection, such as the example in Fig. 7(a), downstream information of $s z_{b_{1}}$ and $l_{1}$ are used. If it 


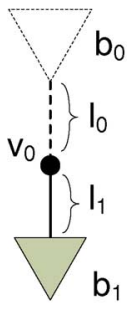

(a)

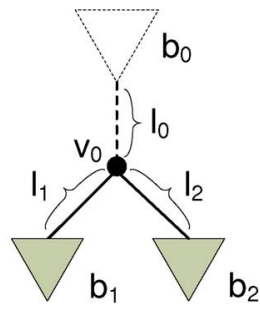

(b)
Fig. 7. Driving length reference. (a) Single wire. (b) Branches.

is a binary branch connection, such as the example in Fig. 7(b), $s z_{b_{1}}, s z_{b_{2}}, l_{1}$, and $l_{2}$ are used. The respective equations to compute $l_{0}$ under these two examples are shown as follows:

$$
l_{0}= \begin{cases}\gamma\left(l_{1}, s z_{b_{1}}\right): & \text { single branch } \\ \gamma\left(l_{1}, l_{2}, s z_{b_{1}}, s z_{b_{2}}\right): & \text { binary branches. }\end{cases}
$$

Notice that for every set of upstream/downstream buffer sizes, denoted as a 3-tuple of $\left(s z_{b_{0}}, s z_{b_{1}}, s z_{b_{2}}\right)$, there is an independent index in the table providing the according driving strength. During each synthesis, buffer size at each insertion point is constant. Wirelength is a real value in the nanometer scale. Therefore, we need to quantize the wirelength of $l_{0}, l_{1}$ and $l_{2}$ during the construction of the slew table, in order to maintain the table within an acceptable scale. $s z_{b_{0}}, s z_{b_{1}}, l_{1}$, and $l_{2}$ are all known values as the input arguments to the lookup function, and the output result of the function is the upstream driving length $l_{0}$. At the clock sinks, the load capacitance is converted into equivalent wire length. From the discrete table records, we assume that the downstream wirelength $l_{1}$ should be in a range of $\left(\operatorname{len}_{\mathrm{HI}}^{D}, \operatorname{len}_{\mathrm{LO}}^{D}\right)$. The corresponding results of upstream driving length are len ${ }_{\mathrm{HI}}$ and $l e \mathrm{n}_{\mathrm{LO}}^{U}$, respectively. The two weighting factors are computed by the following equation:

$$
w_{\mathrm{HI}}=\frac{\operatorname{len}_{\mathrm{HI}}^{D}-l_{1}}{\operatorname{len}_{\mathrm{HI}}^{D}-\operatorname{len}_{\mathrm{LO}}^{D}} \quad w_{\mathrm{LO}}=1-w_{\mathrm{HI}} .
$$

Therefore, the resulting driving length is computed by the following formula:

$$
l_{0}=w_{\mathrm{HI}} \times \operatorname{len}_{\mathrm{HI}}^{U}+w_{\mathrm{LO}} \times \operatorname{len}_{\mathrm{LO}}^{U} .
$$

Notice that the above procedure is based on the single wire connection between buffers of the same or different sizes. In binary branching case with the inclusion of clock gates, similar method can still be applied with very good estimation on the driver ability. We can obtain $l_{0}$ for a binary branch in the same way, as shown in Fig. 7(b). The only difference is that there are two additional indices required for the slew table, which are $s z_{b_{2}}$ and $l_{2}$ as the downstream buffer size and wire length of the other branch.

\section{Fast Information Updating}

During the procedure of bottom-up clock tree synthesis, information updating at every node (sink or internal node)
TABLE I

COMPLEXITY COMPARISON

\begin{tabular}{|l|r|r|}
\hline Synthesizers & Average & Worst Case \\
\hline PACTS,PSACTS & $O\left(B+N^{2}+N K^{2}\right)$ & $O\left(B+N^{3}+N K^{2}\right)$ \\
\hline GCR,GCRred & $O\left(B+N^{2}+N^{2} K^{2}\right)$ & $O\left(B+N^{3}+N^{2} K^{2}\right)$ \\
\hline GBCR,GBCRnt & $O\left(N^{2}+B N\right)$ & $O\left(N^{3}+B N\right)$ \\
\hline
\end{tabular}

TABLE II

CIRCUIT INFORMATION OF THE BENCHMARKS FrOM R1 TO R5

\begin{tabular}{|l|r|r|r|r|r|}
\hline Circuits & $\begin{array}{c}\text { Chip Size } \\
(m m \times m m)\end{array}$ & $\begin{array}{c}\text { No. of } \\
\text { Sinks }\end{array}$ & $\begin{array}{c}\text { No. of } \\
\text { Inst. }\end{array}$ & $\begin{array}{c}\text { Inst. } \\
\text { Length }\end{array}$ & $\begin{array}{c}\text { Average } \\
\text { Activity (\%) }\end{array}$ \\
\hline r1 & $8.60 \times 8.60$ & 267 & 64 & 10000 & 39.86 \\
r2 & $11.6 \times 11.7$ & 598 & 89 & 10000 & 39.60 \\
r3 & $12.2 \times 12.0$ & 862 & 108 & 10000 & 36.91 \\
r4 & $15.9 \times 15.9$ & 1903 & 120 & 10000 & 40.56 \\
r5 & $18.2 \times 17.8$ & 3101 & 160 & 10000 & 37.42 \\
\hline Average & $13.3 \times 13.2$ & 1346.2 & 108.2 & 10000 & 38.87 \\
\hline
\end{tabular}

comprises two components: activity computation and transition probability computation. Let $K$ denote the amount of instructions (number of instruction types), $L$ denote the maximum number of active sinks of all the instructions. $B$ is used to denote the length of the instruction stream and $N$ is used to denote the number of sinks. $B$ instructions are read into the memory in the beginning. Previous work [31] traverses the instruction stream to update the activity of each node with complexity $O(B N)$. This is the bottleneck of run-time when $B$ becomes quite large. This method is quite common in many experiments. Instead, we use the approach of instruction frequency table (IFT) [30] to compute activities. For every instruction, the set of active sinks and the probability are pre-defined. The engaged instruction set of every sink is also pre-defined. We can compute the activities of all the sinks by summing up the probabilities of their engaged instructions with complexity $O(K L)$. The activity of each internal node is computed similarly, based on the number of sinks it contains. As a result, the total complexity of activity computation is $O(N K L)$.

In our work, we propose an improved transition probability computation approach, based on the method of instruction transition-module activation table (ITMAT) [30]. To avoid traverse the instruction stream, a previous work [30] proposed an activation table with $N \times(K \times K)$ entries. This table is constructed so every clock tree node can be stored. The only index for the table is sink $\left(s_{i}\right)$, and each entry contains two groups of $K \times K$ elements based on all possible instruction transitions $\left(I_{i} \rightarrow I_{j}, 1 \leq i \leq K, 1 \leq j \leq K\right)$. These two groups of elements are the probability of transition and the transition tags $(01$, $10,11,00)$. Every node uses bitwise $O R$ operation on the transition tags of all its sinks and computes the sum of transition probability at the tag of 01 or 10 . The computation complexity on each node is $O\left(N K^{2}\right)$. As a result, the total complexity for the whole clock tree becomes $O\left(N^{2} K^{2}\right)$. In our work, we improve the total complexity to $O\left(N K^{2}\right)$. In our approach, we store the activation table of each node in the memory, with the additional memory cost of $N K^{2}$. Notice that the ITMAT of any nodes can be reused by their parent nodes, so the total memory cost will never exceed $N K^{2}$, which is not worth concerning. During the bottom-up clock tree synthesis, we compute the $O R$ result 
TABLE III

Performance Comparison Between PACTS ANd the Other Clock Gating Works

\begin{tabular}{|c|c|c|c|c|c|c|c|c|c|c|c|c|}
\hline \multirow{2}{*}{ Circuits } & \multicolumn{2}{|c|}{ PACTS } & \multicolumn{2}{|c|}{ GCR [30] } & \multicolumn{2}{|c|}{ GCRred [30] } & \multicolumn{2}{|c|}{ GBCR [31] } & \multicolumn{2}{|c|}{ GBCRnt [31] } & \multicolumn{2}{|c|}{ BCTS } \\
\hline & $\mathrm{SC}$ & CPU & $\mathrm{SC}$ & CPU & $\mathrm{SC}$ & CPU & $\mathrm{SC}$ & CPU & $\mathrm{SC}$ & $\mathrm{CPU}$ & $\mathrm{SC}$ & CPU \\
\hline rl & 39.33 & 0.36 & 101.12 & 0.08 & 50.95 & 0.09 & 46.81 & NA & 40.36 & NA & 42.95 & 0.36 \\
\hline $\mathrm{r} 2$ & 84.28 & 0.57 & 276.03 & 0.61 & 115.35 & 0.61 & 98.63 & NA & 85.86 & NA & 88.77 & 0.57 \\
\hline r3 & 112.53 & 0.85 & 405.56 & 1.50 & 157.47 & 1.52 & 141.66 & NA & 121.17 & NA & 120.65 & 0.85 \\
\hline r4 & 241.55 & 2.29 & 1104.28 & 5.81 & 367.08 & 5.90 & 369.28 & NA & 249.82 & NA & 251.61 & 2.29 \\
\hline r5 & 372.19 & 6.14 & 2037.13 & 18.8 & 590.84 & 19.03 & 572.82 & NA & 377.87 & NA & 389.70 & 6.14 \\
\hline Average & 169.98 & 2.04 & 784.28 & 5.36 & 256.34 & 5.43 & 245.84 & $\overline{\mathrm{NA}}$ & 175.02 & NA & 178.74 & 2.04 \\
\hline w.r.t. & 1.00 & 1.00 & 4.61 & 2.63 & 1.51 & 2.66 & 1.45 & NA & 1.03 & NA & 1.05 & 1.00 \\
\hline
\end{tabular}

of the ITMAT of two nodes that are merged. Hence, we build the ITMAT for the new parent node. The complexity of this computation is $O\left(K^{2}\right)$ at each node, and the total complexity of transitional probability computation is $O\left(N K^{2}\right)$.

\section{Complexity Analysis}

The total complexity of our work comprises of four components: activity computation, transition probability computation, instruction stream input and neighbor updating. As discussed in Section III-C, the computation complexity of activity computation and transition probability computation are $O(N K L)$ and $O\left(N K^{2}\right)$, respectively. We read the input instruction stream into memory at the beginning, of which the complexity is $O(B)$. Our topology implementation is NNS [15]. We need to update the neighbor information of all the related nodes after each iteration of merging two nodes to a new one. During each iteration, there are $N$ neighoring computations on average, and up to $N^{2}$ computations in the worst-case. As there are $N$ iterations to construct the clock tree, the complexity of neighbor updating is $O\left(N^{2}\right)$ on average and $O\left(N^{3}\right)$ in the worst case. As a result, the overall complexity of our clock gating work is $O\left(B+N^{2}+\right.$ $\left.N K^{2}+N K L\right)$ for average case. Since $K \times L=O(N)$, we simplify it to be $O\left(B+N^{2}+N K^{2}\right) .\left(B+N^{3}+N K^{2}\right)$ is the complexity of our approach in the worst case.

The average time complexity of GBCR and GBCRnt [31] is reported as $O\left(N^{2}+B N\right)$. The complexity in the worst case is $O\left(N^{3}+B N\right)$. However, the value of $B$ is significantly larger than $K$ and even $K^{2}$. Thus, their complexity is also larger than the complexity of our work. For example, the length of the instruction stream $B$ should be increased for improved accuracy of simulation, for instance, from 10000 to 1000000 while the number of instructions $K$ and the maximum number of active sinks $L$ remains the same. The runtime of GBCR and GBCRnt will be linearly increased by $990000 \times N$. However, there is only a constant addition of 990000 in our approach. The complexity comparison among different works is shown in Table I.

\section{EXPERIMENTAL RESULTS}

The experimental results regarding our clock gating works are presented in this section. We implement our gated clock tree synthesizers (PACTS and PSACTS) in C language and the program is executed on a Linux operating system with an Intel Core2 Quad 2.4 GHz CPU and 4 GB memory.

The benchmark suite ( $\mathrm{r} 1$ to $\mathrm{r} 5$ ) [7] is used to test our synthesizer PACTS. Additionally, the benchmarks were modified in [30] with the inclusion of according activity pattern for every sink. The activity pattern is transformed from the instruction
TABLE IV

Circuit INFORMATION OF THE BENCHMARKS From ISPD [32], [33]

\begin{tabular}{|l|r|r|r|r|r|}
\hline Circuits & $\begin{array}{c}\text { Chip Size } \\
(m m \times m m)\end{array}$ & $\begin{array}{c}\text { No. of } \\
\text { Sinks }\end{array}$ & $\begin{array}{c}\text { No. of } \\
\text { Inst. }\end{array}$ & $\begin{array}{c}\text { Inst. } \\
\text { Length }\end{array}$ & $\begin{array}{c}\text { Average } \\
\text { Activity(\%) }\end{array}$ \\
\hline f11 & $11.0 \times 11.0$ & 121 & 45 & 10000 & 37.24 \\
f12 & $8.10 \times 12.6$ & 117 & 44 & 10000 & 38.97 \\
f21 & $12.6 \times 11.7$ & 117 & 44 & 10000 & 40.45 \\
f22 & $11.7 \times 4.90$ & 91 & 40 & 10000 & 40.02 \\
f31 & $17.1 \times 17.1$ & 273 & 65 & 10000 & 43.62 \\
f32 & $17.0 \times 17.0$ & 190 & 55 & 10000 & 44.40 \\
fnb1 & $2.57 \times 2.12$ & 330 & 71 & 10000 & 41.66 \\
f33 & $15.3 \times 15.3$ & 209 & 57 & 10000 & 36.70 \\
f34 & $16.0 \times 16.0$ & 157 & 48 & 10000 & 43.82 \\
f35 & $15.3 \times 15.3$ & 193 & 55 & 10000 & 35.75 \\
fnb2 & $6.39 \times 4.39$ & 440 & 79 & 10000 & 37.73 \\
\hline Average & $12.1 \times 11.6$ & 203 & 54.8 & 10000 & 40.03 \\
\hline cns01 & $8.00 \times 8.00$ & 1107 & 115 & 10000 & 42.25 \\
cns02 & $13.0 \times 7.00$ & 2249 & 145 & 10000 & 38.56 \\
cns03 & $3.07 \times 0.49$ & 1200 & 117 & 10000 & 35.56 \\
cns04 & $2.13 \times 2.69$ & 1845 & 136 & 10000 & 39.11 \\
cns05 & $2.32 \times 2.55$ & 1016 & 113 & 10000 & 43.21 \\
cns06 & $1.95 \times 0.89$ & 981 & 111 & 10000 & 43.40 \\
cns07 & $2.54 \times 1.45$ & 1915 & 138 & 10000 & 34.26 \\
cns08 & $1.83 \times 1.63$ & 1134 & 115 & 10000 & 43.39 \\
\hline Average & $4.36 \times 3.09$ & 1430.8 & 123.8 & 10000 & 40.03 \\
\hline
\end{tabular}

stream based on the logic description of all the instructions. The information of the benchmarks is shown in Table II. For the whole benchmark suite, the average amount of clock sinks, the average number of instructions $K$, the length of instruction stream $B$ and the average activity are about $1346.2,108.2$, and $38.9 \%$, respectively.

Five previous synthesizers are presented with PACTS for performance comparison. GCR and GCRred [30] were proposed in 2001. GCR handles the gated clock routing algorithm with gate insertion at every internal node of the clock tree. GCRred is an advanced version of GCR, where clock gates are selectively removed for switched capacitance improvement. GBCR and GBCRnt [31] were proposed in 2008. GBCR handles the gated and buffered clock routing with the application of dynamic programming and solution sampling. Meanwhile, the topology generated in GCR is re-used in GBCR. In GBCRnt, a new topology was implemented for performance improvement. Buffered clock tree synthesis (BCTS) is a modified version PACTS with no gate insertion but only buffer insertion. The unit resistance of wire $\left(R_{w}\right)$ is $0.003 \Omega / \mathrm{um}$. The unit capacitance of wire $\left(C_{w}\right)$ is $0.02 \mathrm{fF} / \mathrm{um}$. The loading capacitance of clock gate and buffer $\left(C_{g}\right.$ and $\left.C_{b}\right)$ are $30 \mathrm{fF}$.

The performance of the six clock gating research works are shown in Table III. Switched capacitance (SC) and runtime (CPU) are shown in the table. They are measured in $\mathrm{pF}$ and seconds, respectively. It can be observed that GCR has the largest 
TABLE V

Performance Comparison Between PSACTS and SABCTS Base on the ISPD Benchmark Suite [32], [33]

\begin{tabular}{|l|r|r|r|r|r|r|r|r|}
\hline \multirow{2}{*}{ Circuits } & \multicolumn{4}{|c|}{ PSACTS } & \multicolumn{4}{|c|}{ SABCTS } \\
\cline { 2 - 9 } & \multicolumn{1}{|c|}{ SC } & skew & GT & \multicolumn{1}{c|}{ BF } & SC & skew & GT & BF \\
\hline f11 & 36.74 & 19.44 & 24 & 164 & 40.80 & 19.42 & 0 & 188 \\
f21 & 35.27 & 15.69 & 30 & 151 & 38.38 & 17.74 & 0 & 181 \\
f22 & 39.95 & 18.73 & 25 & 169 & 42.72 & 18.56 & 0 & 194 \\
f31 & 24.20 & 18.31 & 20 & 117 & 26.51 & 13.97 & 0 & 127 \\
f32 & 84.78 & 24.58 & 28 & 393 & 88.12 & 23.46 & 0 & 421 \\
fnb1 & 64.18 & 22.41 & 23 & 281 & 66.36 & 21.60 & 0 & 304 \\
f33 & 15.86 & 16.06 & 69 & 34 & 16.70 & 16.02 & 0 & 103 \\
f34 & 61.49 & 20.14 & 25 & 296 & 67.67 & 19.98 & 0 & 321 \\
f35 & 54.00 & 16.72 & 18 & 242 & 56.10 & 16.97 & 0 & 260 \\
fnb2 & 60.14 & 19.02 & 22 & 287 & 64.03 & 18.95 & 0 & 309 \\
\hline Average & 29.79 & 24.07 & 54 & 124 & 31.21 & 24.01 & 0 & 178 \\
\hline w.r.t. & 1.00 & 19.56 & 31 & 205 & 48.96 & 19.15 & 0 & 235 \\
\hline cns01 & 82.79 & 26.40 & 78 & 397 & 83.68 & 24.61 & 0 & 470 \\
cns02 & 156.20 & 33.69 & 152 & 770 & 159.27 & 33.92 & 0 & 922 \\
cns03 & 31.76 & 18.81 & 135 & 75 & 33.19 & 19.77 & 0 & 210 \\
cns04 & 37.72 & 18.88 & 106 & 117 & 37.74 & 19.06 & 0 & 223 \\
cns05 & 16.88 & 15.99 & 25 & 83 & 17.27 & 16.08 & 0 & 108 \\
cns06 & 25.83 & 28.68 & 126 & 54 & 27.89 & 28.62 & 0 & 180 \\
cns07 & 41.42 & 21.55 & 134 & 147 & 43.01 & 21.52 & 0 & 281 \\
cns08 & 27.84 & 21.99 & 131 & 53 & 28.78 & 21.95 & 0 & 184 \\
\hline Average & 52.49 & 23.25 & 111 & 212 & 53.86 & 23.19 & 0 & 322 \\
\hline w.r.t. & 1.00 & 1.00 & 1.00 & 1.00 & 1.03 & 0.99 & 0.00 & 1.52 \\
\hline
\end{tabular}

switched capacitance. It means that the corresponding clock trees have the largest power consumption. The major reason is that every node in GCR is assigned with a clock gate. In this case, the resultant controller tree becomes the main contribution to the total power usage. With the inclusion of gate removal technique, the resulting cost of the switched capacitance in GCRred is reduced by $67.3 \%$ compared to GCR. In spite of the performance improvement, the heuristic of gate removing in GCRred is generally based on the activity comparison, which is not fully performance-driven. There are still some redundant gate insertions which may cause negative effect towards the power reduction. GBCRnt is an improved version of GBCR, where a new topology is devised. It is also based on NNS [15] with newly developed cost function involved. With the inclusion of the new topology, GBCRnt has improvement on both the clock tree and the controller tree. Compared to GBCR, the resultant total switched capacitance is reduced by $28.8 \%$.

Our gate insertion method applied in PACTS is consistent with the result of the pairing cost, which can further enhance the performance of the topology generation. Compared to the best reported experimental results from GBCRnt, the resulting total switched capacitance is smaller consistently for all test cases. This is because the efficiency of each gate for turning off idle branches is higher in our synthesizer. The results of buffered clock tree synthesis (denoted as BCTS) are also listed in the Table III. The same cost function in (8) is applied for topology implementation. The slew constraint $\left(C_{d} \leq 20 \times C_{g}\right)$ is applied but only buffers can be inserted. The switched capacitance of BCTS is larger than the switched capacitance of PACTS. It means that clock tree synthesis with appropriate insertion of clock gates can reduce the dynamic power consumption significantly. In general, experimental results show that our power aware clock tree synthesizer, PACTS, can outperform all the other clock gating works with the minimum switched capacitance in every benchmark.
For the comparison of runtime, we execute the programs of GCR and GCRred in the same platform as PACTS. In Table III, the CPU runtime of PACTS, GCR, and GCRred are listed. Although PACTS may have longer runtime (because of memory initialization) in some smaller test cases, experimental results show that the average runtime is reduced by $61.9 \%$ compared to the runtime of GCR and GCRred. It is because the complexity of PACTS has been reduced from $O\left(B+N^{2} K^{2}\right)$ to $\left(B+N^{2}+\right.$ $\left.N K^{2}\right)$ in average. The runtime of GBCR and GBCRnt have not been obtained for comparison. Based on the comparison of complexity, PACTS should be better than GBCR and GBCRnt in terms of runtime.

In order to test the power and slew aware clock tree synthesizer, PSACTS, the benchmark suite proposed in the clock network synthesis contest of ISPD 2009 [32] and ISPD 2010 [33] are used. The RC model of wire, buffer and clock gate are also obtained from this contest [32]. We compute the resultant switched capacitance for each benchmark. Additionally, SPICE simulation is applied based on the gated clock routing result for skew estimation and slew verification. The benchmark information is shown in Table IV. The unit resistance of the wire is $0.0003 \Omega / \mathrm{nm}$, and the unit capacitance of the wire is 0.00016 $\mathrm{fF} / \mathrm{nm}$. The input capacitance of buffer/gate is $35 \mathrm{fF}$. The cost of parasitic capacitance is removed in this experiment so that the setting is consistent with the previous clock gating experiments on the computation of switched capacitance.

The experimental results are shown in Table V. It is composed of the switched capacitance ( $\mathrm{SC}$ in $\mathrm{pF}$ ), the simulated clock skew by SPICE (skew in ps), the number of clock gates (GT) and buffers (BF). PSACTS is our slew aware clock tree synthesizer. We cannot obtain the result from previous clock gate works because clock slew violation causes un-reasonable clock skew (larger than a few hundreds ps) during simulation. SABCTS (slew-aware buffered clock tree synthesizer) is similar to PSACTS but only the buffers can be inserted to reduce 
the clock slew and there is no insertion of the clock gates. By comparing PSACTS with SABCTS, the tradeoff between clock skew and the switched capacitance can be illustrated. By applying the insertion of clock gate, the switched capacitance can be reduced in every benchmark. The number of clock sinks is smaller in ISPD09 benchmarks. The resultant switched capacitance of PSACTS is reduced by $5.98 \%$ in average compared to the result of SABCTS. The clock skew of PSACTS is increased by only $0.4 \mathrm{ps}$ in average. For the ISPD 10 benchmarks, the resultant switched capacitance of PSACTS is reduced by $2.60 \%$ in average compared to the result of SABCTS. The clock skew of PSACTS is basically the same as the skew of SABCTS. The clock skew of PSACTS is increased by only $0.06 \mathrm{ps}$ in average. If the larger penalty on clock skew is allowed in PSACTS, the switched capacitance can be reduced significantly.

\section{CONCLUSION}

In this paper, two clock tree synthesizers, PACTS and PSACTS are proposed. Comparing with previous clock gating works, several novel methods are introduced. They include the power aware topology generation, the concurrent gate insertion and the fast information updating. From the experimental results, it is shown that PACTS has good performance (smaller switched capacitance) and high efficiency (shorter runtime). In addition, PSACTS is also developed to satisfy the concrete slew constraint. The experimental results show that it can reduce the switched capacitance with satisfying the slew constraint. In conclusion, our proposed clock tree synthesizers have better performance and improved efficiency, and it can be applied more practically in the industry.

\section{REFERENCES}

[1] J. Cong, A. B. Kahng, C.-K. Koh, and C.-W. A. Tsao, "Bounded-skew clock and steiner routing," ACM Trans. Design Autom. Electron. Syst., vol. 3, pt. 3, pp. 341-388, 1998.

[2] T. Kitahara, F. Minami, T. Ueda, K. Usami, S. Nishio, M. Mruakata, and T. Mitsuhashi, "A clock-gating method for low-power LSI design," in Proc. Asia South Pacific Design Autom. Conf., 1998, pp. 307-312.

[3] M. A. B. Jackson, A. Srinivasan, and E. S. Kuh, "Clock routing for high-performance ICs," in Proc. IEEE/ACM Design Autom. Conf., 1990 , pp. $573-579$

[4] M. Edahiro and T. Yoshimura, "Minimum path-length equi-distant routing," in Proc. IEEE Asia-Pacific Conf. Circuits Syst., 1992, pp. $41-46$.

[5] A. Kahng, J. Cong, and G. Robins, "High-performance clock routing based on recursive geometric matching," in Proc. IEEE/ACM Design Autom. Conf., 1991, pp. 322-327.

[6] W. C. Elmore, "The transient response of damped linear networks with particular regard to wide band amplifiers," J. Appl. Phys., vol. 19, no. 1, pp. 55-63, Jan. 1948.

[7] R.-S. Tsay, "Exact zero skew," in Proc. IEEE/ACM Int. Conf. Comput.Aided Design, 1991, pp. 336-339.

[8] K. D. Boese and A. B. Kahng, "Zero-skew clock routing trees with minimum wirelength," in Proc. 5th Annu. IEEE Int. ASIC Conf. Exhib., 1992, pp. 17-21.

[9] T. H. Chao, Y. C. Hsu, J. M. Ho, and A. B. Kahng, "Zero skew clock routing with minimum wirelength," IEEE Trans. Circuits Syst. II, Analog Digit. Signal Process., vol. 39, no. 11, pp. 799-814, Nov. 1992.

[10] L. P. P. P. van Ginneken, "Buffer placement in distributed RC-tree networks for minimal elmore delay," in Proc. Int. Symp. Circuits Syst., 1990 , pp. 865-868.

[11] J. D. Cho and M. Sarrafzadeh, "A buffer distribution algorithm for high-speed clock routing," in Proc. IEEE/ACM Design Autom. Conf., 1993, pp. 537-543.
[12] W. Shi and Z. Li, "A fast algorithm for optimal buffer insertion," IEEE Trans. Comput.-Aided Design Integr. Circuits Syst., vol. 24, no. 6, pp. 79-891, May 2005.

[13] A. Vittal and M. Marek-Sadowska, "Low power buffered clock tree design," IEEE Trans. Comput.-Aided Design Integr. Circuits Syst., vol. 16, no. 9, pp. 965-975, Sep. 1997.

[14] G. E. Tellez and M. Sarrafzadeh, "Minimal buffer insertion in clock trees with skew and slew rate constraints," IEEE Trans. Comput.-Aided Design Integr. Circuits Syst., vol. 16, no. 4, pp. 333-342, Apr. 1997.

[15] M. Edahiro, "A clustering-based optimization algorithm in zero-skew routings," in Proc. IEEE/ACM Design Autom. Conf., 1993, pp. 612-616.

[16] R. Chaturvedi and J. Hu, "Buffered clock tree for high quality IC design," in Proc. Int. Symp. Quality Electron. Design, 2004, pp. 381-386.

[17] J. Lu, W. K. Chow, C. W. Sham, and E. F. Y. Young, "A dual-MST approach for clock network synthesis," in Proc. Asia South Pacific Design Autom. Conf., 2010, pp. 467-473.

[18] Y.-Y. Chen, C. Dong, and D. Chen, "Clock tree synthesis under aggressive buffer insertion," in Proc. IEEE/ACM Design Autom. Conf., 2010 , pp. 86-89.

[19] W.-H. Liu, Y.-L. Li, and H.-C. Chen, "Minimizing clock latency range in robust clock tree synthesis," in Proc. Asia South Pacific Design Autom. Conf., 2010, pp. 389-394.

[20] C. M. Chang, S. H. Huang, Y. K. Ho, J. Z. Lin, H. P. Wang, and Y. S. Lu, "Type-matching clock tree for zero skew clock gating," in Proc. IEEE/ACM Design Autom. Conf., 2008, pp. 714-719.

[21] M. Donno, A. Ivaldi, L. Benini, and E. Macii, "Clock-tree power optimization based on RTL clock-gating," in Proc. IEEE/ACM Design Autom. Conf., 2003, pp. 622-627.

[22] Y. Luo, J. Yu, J. Yang, and L. Bhuyan, "Low power network processor design using clock gating," in Proc. IEEE/ACM Design Autom. Conf., 2005, pp. 712-715.

[23] H. Li, S. Bhunia, Y. Chen, K. Roy, and T. N. Vijaykumar, "DCG: Deterministic clock-gating for low-power microprocessor design," IEEE Trans. Very Large Scale Integr. (VLSI) Syst., vol. 12, no. 3, pp. 245-254, Mar. 2004.

[24] D. Garrett, M. Stan, and A. Dean, "Challenges in clockgating for a low power ASIC methodology," in Proc. Int. Symp. Low Power Electron. Design, 1999, pp. 176-181.

[25] W. Shen, Y. Cai, X. Hong, and J. Hu, "An effective gated clock tree design based on activity and register aware placement," IEEE Trans. Very Large Scale Integr. (VLSI) Syst., vol. 18, no. 12, pp. 1639-1648, Dec. 2010.

[26] G. E. Tellez, A. Farrahi, and M. Sarrafzadeh, "Activity-driven clock design for low power circuits," in Proc. IEEE/ACM Int. Conf. Comput.Aided Design, 1995, pp. 62-65.

[27] A. H. Farrahi, C. Chen, A. Srivastava, G. Tellez, and M. Sarrafzadeh, "Activity-driven clock design," IEEE Trans. Comput.-Aided Design Integr. Circuits Syst., vol. 20, no. 6, pp. 705-714, Jun. 2001

[28] C. Chen, C. Kang, and M. Sarrafzadeh, "Activity-sensitive clock tree construction for low power," in Proc. Int. Symp. Low Power Electron. Design, 2002, pp. 279-282.

[29] J. Oh and M. Pedram, "Gated clock routing minimizing the switched capacitance," in Proc. Design, Autom. Test in Euro. Conf. Exhib., 1998, pp. 692-697.

[30] J. Oh and M. Pedram, "Gated clock routing for low-power microprocessor design," IEEE Trans. Comput.-Aided Design Integr. Circuits Syst., vol. 20, no. 6, pp. 715-722, Jun. 2001.

[31] W. C. Chao and W. K. Mak, "Low-power gated and buffered clock network construction," ACM Trans. Design Autom. Electron. Syst., vol. 13, no. 1, pp. 20:1-20:20, Jan. 2008.

[32] C. N. Sze, P. Restle, G.-J. Nam, and C. Alpert, "ISPD2009 clock network synthesis contest," in Proc. ACM Int. Symp. Phys. Design, 2009, pp. $149-150$.

[33] C. N. Sze, "ISPD 2010 high performance clock network synthesis contest: Benchmark suite and results," in Proc. ACM Int. Symp. Phys. Design, 2010, pp. 149-150.

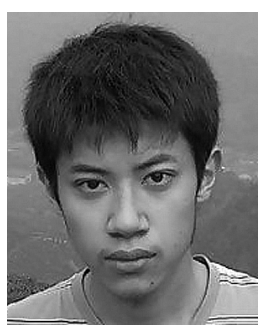

Jingwei Lu received the Bachelor degree from Zhejiang University, China, in 2008, and the M.Phil. degree from The Hong Kong Polytechnic University, Hong Kong, in 2010 . He is currently pursuing the $\mathrm{Ph}$.D. degree from the University of California, San Diego.

His research interests include design automation of VLSI including placement and clock planning. 


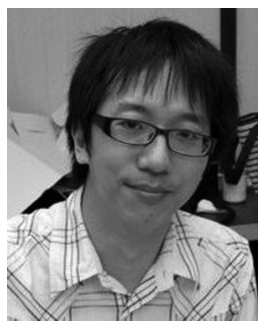

Wing-Kai Chow received the Bachelor degree from The Hong Kong Polytechnic University, Hong Kong, in 2009.

He was a Research Assistant with The Hong Kong Polytechnic University in 2010. Currently, he works as a Research Assistant with The Chinese University of Hong Kong. His research interests include design automation of VLSI such as routing and clock planning.

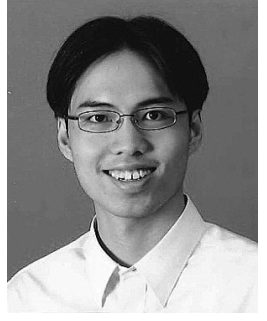

Chiu-Wing Sham received the Bachelor degree in computer engineering and the M.Phil. degree and the $\mathrm{Ph} . \mathrm{D}$. degree from The Chinese University of Hong Kong, Hong Kong, in 2000, 2002, and 2006, respectively.

He was a Research Engineer with Synopsys, Shanghai, China, and an Electronic Engineer on the FPGA applications of motion control system with ASM, Hong Kong. He was a Lecturer in The Hong Kong Polytechnic University since 2006. His research interests include design automation of VLSI such as floorplanning, placement and clock planning, and digital design. 